\title{
BMJ Open Implementing shared decision-making in interprofessional home care teams (the IPSDM-SW study): protocol for a stepped wedge cluster randomised trial
}

France Légaré, ${ }^{1,2}$ Nathalie Brière, ${ }^{3}$ Dawn Stacey, ${ }^{4,5}$ Guy Lacroix, ${ }^{6}$ Sophie Desroches, ${ }^{1,7}$ Serge Dumont, ${ }^{6,7,8}$ Kimberly D Fraser, ${ }^{9}$ Louis-Paul Rivest, ${ }^{10}$ Pierre J Durand, ${ }^{2,3}$ Stéphane Turcotte, ${ }^{1}$ Monica Taljaard, ${ }^{4,5,6,7,8,9,10,11}$ Henriette Bourassa, ${ }^{12}$ Lise Roy, ${ }^{12}$ Geneviève Painchaud Guérard ${ }^{1}$

To cite: Légaré $\mathrm{F}$, Brière $\mathrm{N}$, Stacey D, et al. Implementing shared decision-making in interprofessional home care teams (the IPSDM-SW study): protocol for a stepped wedge cluster randomised trial. BMJ Open 2016;6: e014023. doi:10.1136/ bmjopen-2016-014023

- Prepublication history for this paper is available online. To view these files please visit the journal online (http://dx.doi.org/10.1136/ bmjopen-2016-014023).

Received 25 August 2016 Revised 21 October 2016 Accepted 27 October 2016

CrossMark

For numbered affiliations see end of article.

Correspondence to Dr France Légaré; france. legare@mfa.ulaval.ca

\section{ABSTRACT}

Introduction: The frail elderly in Canada face a tough decision when they start to lose autonomy: whether to stay at home or move to another location. This study seeks to scale up and evaluate the implementation of shared decision-making (SDM) in interprofessional (IP) home care teams caring for elderly clients or their caregivers facing a decision about staying at home or moving elsewhere.

Methods: A stepped wedge cluster randomised trial involving 8 Health and Social Service Centers (HSSCs) will be conducted with IP home care teams. HSSCs are the unit of randomisation. A decision guide will be passively distributed to all of the participating HSSCs at the beginning of the project. The participating HSSCs will then be randomised to 1 of 4 intervention start times, separated by 7-month intervals. The primary outcome is whether or not clients and caregivers assumed an active role in decisionmaking, assessed with a modified version of the Control Preferences Scale. The intervention, targeted at IP home care teams, consists of a 1.5 hour online tutorial and a 3.5 hour skills building workshop in IP SDM. Clients will be eligible for outcome assessment if they (1) are aged $\geq 65$; (2) are receiving care from the IP home care team of the enrolled HSSCs; (3) have made a decision about whether to stay at home or move to another location during the recruitment periods; (4) are able to read, understand and write French or English; (5) can give informed consent. If clients are not able to provide informed consent, their primary caregiver will become the eligible participant.

Ethics and dissemination: Ethics committee review approval has been obtained from the Multicenter Ethics Committee of CISSS-Laval. Results will be disseminated at conferences, on websites of team members and in peer-reviewed and professional journals intended for policymakers and managers.

Trial registration number: NCT02592525, Pre-results.

\section{Strengths and limitations of this study}

- This trial addresses a difficult decision for the elderly: whether to stay home or move to another location. This decision is frequently encountered by home care teams.

- By the end of this trial, all enrolled clusters will have been exposed to the intervention.

- The novel stepped wedge design reconciles the need for robust evaluations with political or logistical constraints.

- Recruiting elders and busy providers may be challenging.

- It may be challenging for enrolled clusters to follow the randomisation schedule strictly.

\section{INTRODUCTION}

In the context of home care in Canada, one of the important decisions the frail elderly must face is whether to remain at home (with or without assistance) or move to another location. ${ }^{1}$ The care setting has a direct association with a wide array of outcomes impacting negatively on health, notably depression, pain, pressure ulcers and falls. ${ }^{1}$ In other words, where elderly people live is an important determinant of health. ${ }^{2}$ As older persons with better self-perceived health have lower mortality, ${ }^{3}{ }^{4}$ feelings of control over healthcare decisions ought to play a part in the decision about where they live. Often, this decision is poorly or insufficiently planned with clients and caregivers receiving little or no decision support. ${ }^{5}$ Involving frail elderly clients and caregivers in this decision presents particular challenges as it is often associated with emotional turmoil. ${ }^{6-9}$ Shared decision-making (SDM) tools, such as patient decision aids, increase 
the involvement of patients in decisions about their care $^{10}$ and can improve agreement between patients and their healthcare professionals. ${ }^{11}$ However, little is known about the most effective strategy to promote the use of decision tools in clinical practice or, ultimately, how to scale up SDM across the health and social care system. An interprofessional (IP) approach to SDM is especially relevant to caring for the frail elderly, as chronic illness often means that several different kinds of healthcare providers are involved in their care. Intentional interaction as a team enables healthcare providers to collaboratively support clients in facing difficult decisions, meet their decisional needs and reach healthcare choices that are agreed on by the client, family members/caregivers and the IP team. ${ }^{12}{ }^{13}$ However, multiple barriers to achieving SDM have been identified that are specifically associated with multidisciplinary care settings, such as lack of visibility, lack of trust in expertise of other disciplines and lack of communication between disciplines. ${ }^{14}$ Therefore, training home care teams in SDM using an IP approach that addresses these barriers should facilitate effective uptake of decision tools in clinical practice and ultimately, scale up the implementation of SDM across the health and social care system. The objective of this study is to evaluate the impact of a training programme in IP-SDM (interprofessional approach to SDM) on the proportion of clients and caregivers who report taking an active part in the decision-making process, compared with the passive dissemination of a decision guide. Passive dissemination of printed educational material may have a small beneficial effect on professional practice outcomes, but its effectiveness compared with that of printed educational material as part of a multifaceted intervention is uncertain. ${ }^{15}$ For this purpose, a cross-sectional stepped wedge cluster randomised trial (cRT) is proposed. The null hypothesis is that the addition of a training programme in IP-SDM to the passive dissemination of a decision guide will not increase the proportion of clients and caregivers reporting an active role in the decision-making process.

\section{METHODS AND ANALYSIS}

Study setting and design

Stepped wedge cRTs are particularly well suited for evaluating interventions during implementation into routine practice and in situations in which there is a prior belief that the intervention (SDM training of IP home care teams) will do more good than harm, rather than a prior belief that there is equipoise (harms and benefits are equally balanced). ${ }^{16}$ This stepped wedge cRT will be conducted in the province of Quebec, Canada, with IP home care teams of eight Health and Social Services Centers (HSSCs), which are the units of randomisation. A decision guide will be passively distributed to all participating HSSCs at the beginning of the project. The HSSCs will then be randomised to one of four steps with a total of five data collection phases (figure 1). By the end of the study, all HSSCs will have received the intervention. Data will be collected on different (cross-sectional) samples of clients and caregivers at each data collection phase, but the same IP teams and providers will be involved throughout the trial.

\section{Eligibility criteria}

All HSSCs of Quebec province, which are known in French as Centres intégrés de santé et de services sociaux (CISSS) or Centres intégrés universitaires de santé et de services sociaux (CIUSSS), will be eligible ${ }^{17}$ unless they have participated in an earlier cRT that compared the impact of training home care teams in SDM and provided them with a decision guide during training to usual care. ${ }^{18} \mathrm{We}$ will contact the managers of the home care teams of all eligible HSSCs in random sequence to present the project and ask them about their interest in participating in the study. Clients of participating sites will be eligible for data collection if they: (1) are aged $\geq 65$; (2) are receiving care from the IP home care team of the enrolled HSSC; (3) have made a decision about whether to stay at home or move to another location during the recruitment periods; (4) are able to read, understand and write French or English; (5) can give informed consent. In the case of clients who are not able to provide informed consent, their informal caregiver will become the eligible participant. Caregivers are defined in this study as close relatives or friends. Healthcare professionals of participating IP teams will also be recruited as participants.

\section{Passive dissemination of the decision guide (control period)}

At the beginning of the project, we will ask managers of all enrolled HSSCs to distribute a decision guide to their IP home care teams to be used with their clients and caregivers (passive dissemination). We will offer managers and healthcare professionals as many decision guides as they need, on request.

\section{Intervention}

All IP home care teams will receive the multifaceted intervention at different time points (figure 2). The intervention consists of (1) a 1.5 hour online tutorial, based on the Ottawa Decision Support Tutorial, ${ }^{19}{ }^{20}$ and (2) a 3.5 hour skills building workshop which includes a lecture, a video demonstrating SDM in the context of an IP home care team and performance feedback during a role play. ${ }^{21}$ The online tutorial is completed individually. The intervention will be delivered at the sites allocated to the intervention step. The decision guide distributed before the intervention will still be available in sufficient quantities after the intervention.

Allocation of participating sites to intervention groups The unit of randomisation will be the HSSC responsible for the IP home care teams. Eight HSSCs will be 


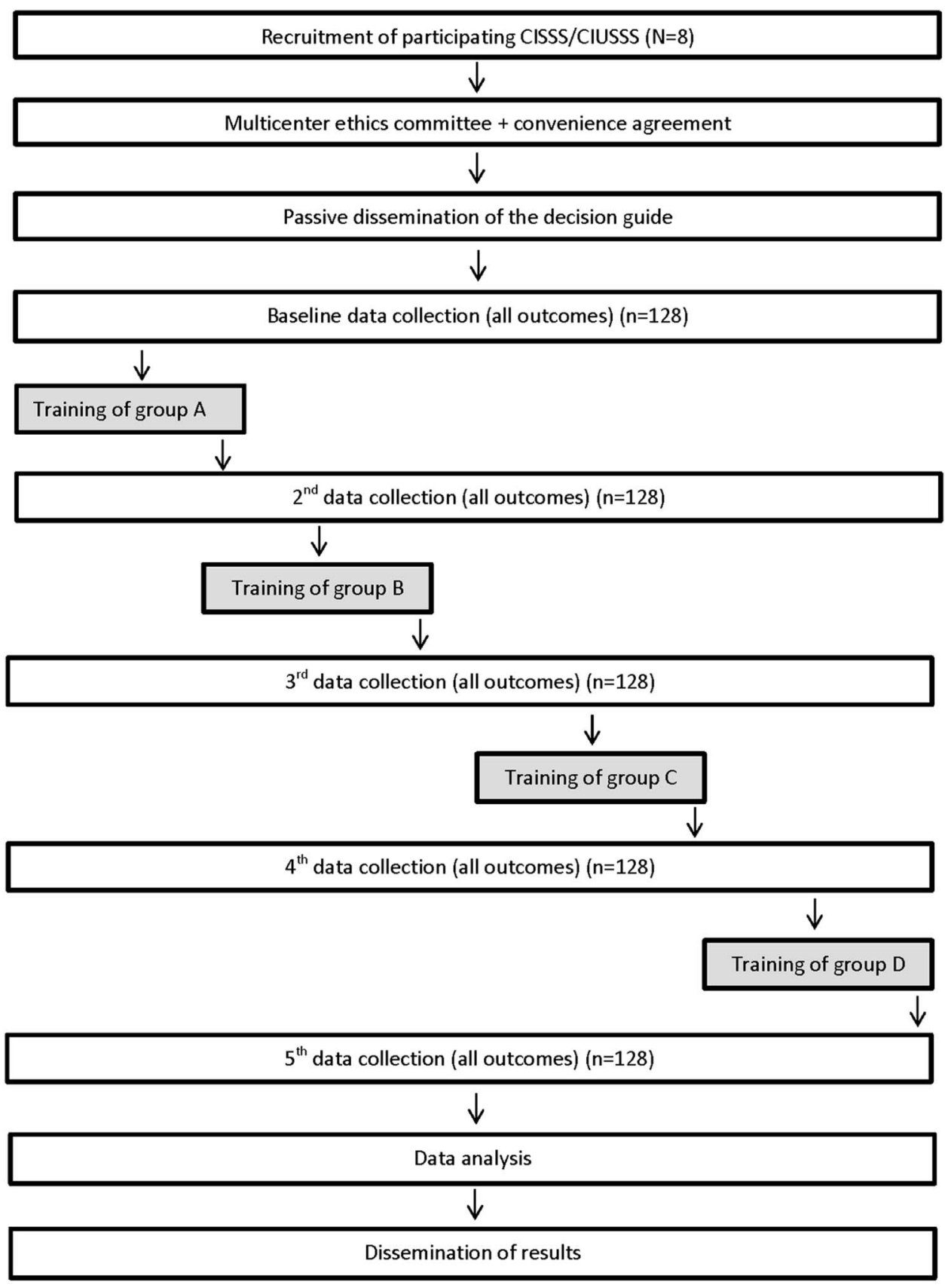

Figure 1 Study flow diagram.

randomised to one of four steps, representing different start times for the intervention, namely at 4 (T4), 11 (T11), 18 (T18) and 25 (T25) months after the start of the baseline data collection (T0) (figure 2). An independent biostatistician will perform randomisation using computer-generated numbers. Allocation will be concealed from investigators involved in the study. The biostatistician performing the allocation will not be involved in data analysis.

\section{Proposed frequency and duration of follow-up}

There will be a total of five data collection periods with 1 month for the intervention between each of them at T4, T11, T18 and T22 (figure 2). Thus, the total duration of data collection will be 32 months. Every 7 months, the intervention will 'step up' to the next group of HSSCs, while the previous groups continue to apply their newly acquired skills, until all four groups have received the intervention (figure 2). As outcome assessments of the trial can only take place once the decision guide is available for use, the decision guide will be passively disseminated to each cluster 3 weeks before the baseline data collection.

Recruitment and loss to follow-up

We will assign trained research assistants (RAs) to each participating site for data collection. The HSSC will be responsible for identifying and contacting eligible 
Figure 2 Stepped wedge study design.

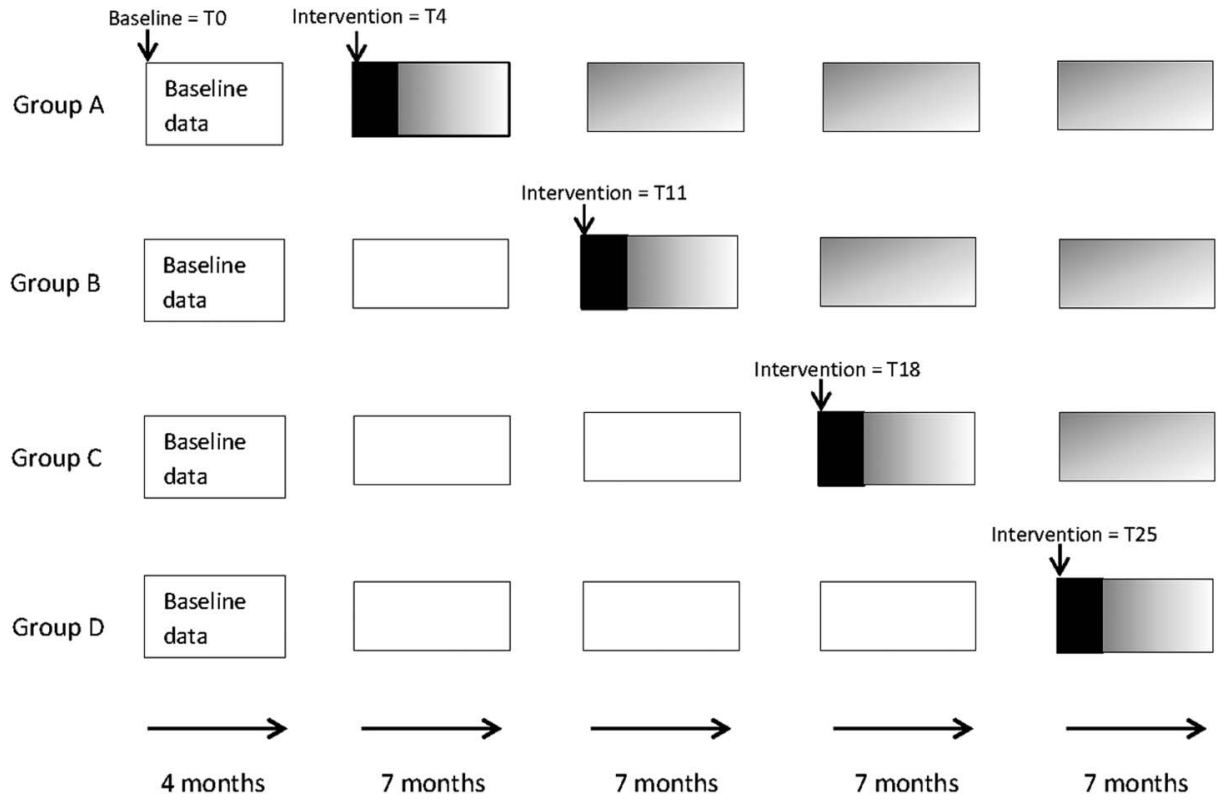

Blank cells $=$ control periods (pre-intervention) + data collection; Shaded cells $=$ postintervention periods + data collection; Black cells $=$ intervention time + no data collection. clients and caregivers and asking permission for the RA to contact them. Their agreement will indicate their interest in participating in the project. Then, the RA will meet interested participants at their home, complete informed consent and proceed with data collection. Healthcare professionals of the IP home care teams will provide informed consent prior to the training session. To facilitate engagement, the research study budget will cover all fees the HSSC may incur in relation to the project. Owing to the nature of this study, which entails no follow-up of participants for the main outcome (only one data collection point for each participant), we expect no loss to follow-up at the clients and caregivers levels.

\section{Outcomes and measures}

The primary outcome will be the role assumed in decision-making by clients and caregivers regarding whether the clients have to stay at home or move to another location. To assess the proportion of clients and caregivers reporting an active role, we will use the modified version of the Control Preferences Scale ${ }^{22}$ designed to assess the role assumed in the decision-making process. ${ }^{23}$ This single-question scale is most frequently used in studies assessing the implementation of SDM in clinical practice. ${ }^{24}$ Response options are: (A) I made the decision; (B) I made the decision after seriously considering my providers' opinion; (C) My providers and I shared the responsibility for making the decision; (D) My providers made the decision after seriously considering my opinion; (E) My providers made the decision. A and $\mathrm{B}$ represent a client or caregiver-controlled decisionmaking process, $\mathrm{C}$ represents an SDM process, and D and $\mathrm{E}$ represent a provider-controlled decision-making process. ${ }^{23}$ For the primary outcome, we will collapse these five categories into just two: A, B and C will represent an active role in the decision-making process and $\mathrm{D}$ and $\mathrm{E}$ will represent a passive role. The modified version of the Control Preferences Scale is used to reduce the burden on frail elderly participants, as suggested by our stakeholders.

Secondary outcomes assessed in caregivers and clients will be (1) their involvement in decision-making, assessed with the D-OPTION scale, a 12-item instrument that assesses 12 specific SDM behaviours during decision-making; ${ }^{25}{ }^{26}$ (2) preferred and chosen option (remain at home or move to another location); (3) decisional conflict, assessed with the 16-item Decisional Conflict Scale; ${ }^{27} 28$ (4) decision regret, assessed with the 5-item Decision Regret Scale; ${ }^{29}$ (5) uptake of the decision guide, assessed by showing an image of the decision guide and asking the question 'Were you shown this decision guide?'; (6) health-related quality of life (only in client), assessed with two subscales (social isolation and emotional reactions) from the Nottingham Health Profile, ${ }^{30-33}$ and (7) burden of care (only in caregivers), assessed with the Zarit Burden Inventory scale (ZBI) ${ }^{34-36}$ Healthcare professionals' behavioural intention to engage into SDM will be measured before and after the intervention with a 12-item theory-based instrument assessing the impact of continuing professional development. ${ }^{37}$ Tutorial and workshop also include an evaluation component. In addition, we will collect qualitative data on the research process by the use of RAs' logbooks in which participants' comments and reactions will be recorded. We will also periodically contact site managers and research agents to solicit their views on the research process. 
Protection against sources of bias and data management

Given the sequential nature of this trial, the investigators and project coordinator will know which HSSCs are receiving the intervention and therefore will not be blinded to group allocation. However, the allocation list will be concealed from the research team for as long as possible; when the next HSSCs group needs to schedule the intervention, only those randomised in that step will be revealed. Also, to minimise sources of bias: (1) we will respect strict concealment of allocation; (2) we will ensure that data collection forms and packages look the same for all groups and data collection periods; (3) two independent data clerks will use a secret group code to perform double data entry; (4) the biostatistician, who will not be involved with randomisation, will be blinded for data analysis as the database will not identify the names of the HSSCs; (5) RAs who collect data from clients and caregivers will be blinded to the allocation status of the HSSC and will be asked not to discuss this information with any participants; they will moreover meet clients and caregivers at their homes or talk to them on the phone); (6) the information given to participants will be the same in the control and intervention periods, and will not refer to the intervention; and (7) analysis will be by intention-to-treat. Questionnaires will be verified by the project coordinator immediately after completion to minimise missing data. Recruitment of clients will be sequential and will be the responsibility of the research team. As HSSC-based home care teams do not share clients and are geographically separated, we do not expect any contamination of the intervention among providers in participating HSSCs. We will emphasise to participants the importance of not sharing the information and material provided with their colleagues from other HSSCs.

\section{Statistical analysis}

\section{Sample size}

The primary outcome is the proportion of clients and caregivers who report an active role in the decisionmaking process. The sample size estimate is informed by preliminary data from another study titled DOLCE. ${ }^{18}$ We used the method developed by Hussey and Hughes for stepped wedge designs. ${ }^{38} 39$ We assumed an average of eight clients and eight caregivers per HSSC in each 6 -month data collection period and an ICC of $0.05 .^{40-44}$ To detect an absolute improvement of $20 \%$ in the primary outcome (from $70 \%$ to $90 \%$ ) with $80 \%$ power using a stepped wedge design with four steps and a twosided test at the $5 \%$ significance level, a total of eight clusters is required (ie, a total of 320 clients and 320 caregivers).

\section{Analysis plan}

We will calculate descriptive statistics of organisational (HSSC) and sociodemographic characteristics for clients, caregivers and healthcare providers. All primary and secondary outcomes will be analysed using the approach described by Hussey and Hughes, ${ }^{38}$ using the 'intention-to-treat' principle. The unit of analysis will be the client (or caregiver). Dichotomous outcomes will be analysed using multilevel logistic regression analysis, while continuous outcomes will be analysed using multilevel linear regression analysis. Time will be modelled as a fixed categorical variable, while the HSSC will be modelled as a random effect to account for the intracluster correlation. ${ }^{38}$ For each outcome analysed, goodness of fit will be assessed and the validity of the underlying assumptions of the model will be checked. All analyses will be conducted using SAS statistical software V.9.4 (SAS Institute, Cary, North Carolina, USA).

\section{Data monitoring}

The main co-PIs (FL, DS and NB) and the project coordinator (GPG) will form a monitoring committee which will provide regular guidance throughout the project (meetings once per month). A trial steering committee made up of co-PIs (FL, DS, NB), co-Is (GL, SDe, SDu, KDF, L-PR and PJD), the project coordinator (GPG) and two caregivers representatives (LR, HB) will also provide regular expert feedback for project monitoring (meetings once every 4 months).

\section{DISCUSSION}

This trial will address challenges and knowledge gaps in the implementation of SDM. There is a growing number of ageing Canadians who are facing the decision regarding location of care and who need client-centred decision support. Hence, there is an urgent need to improve the decision-making process via training of IP home care teams in SDM, as it is not implemented yet in clinical practice. ${ }^{45}$ Therefore, in this study, we seek to scale up the implementation of an (SDM) intervention to IP home care teams in eight HSSCs working with hundreds of clients and caregivers facing decisions about the location of care, and to evaluate the impact of this intervention on the uptake of SDM as indicated by the role assumed by clients and caregivers in the decision-making process. Our results will also enhance the knowledge base about effective interventions for scaling up evidence-based practice across multiple clinical settings by increasing knowledge in the following high-priority research areas: (1) designing health services and supportive policies that meet the health needs of older adults; (2) evaluating innovative and integrated models of primary and community care; (3) empowering patients/self-management/patient experience; (4) supporting caregivers; (5) scaling up evidence-based innovation and (6) advancing decision support and population health monitoring.

\section{DISSEMINATION}

This trial is registered at clinicaltrials.gov (NCT02592525). All participants (clients, caregivers and healthcare professionals) will sign consent forms approved by the ethics 
boards of their respective institutions. All authors will contribute to the dissemination of study results, including caregiver representatives on the trial steering committee. We will tailor effective knowledge translation strategies for each targeted user group (eg, policymakers, clinicians, healthcare organisation managers, seniors' associations). We will disseminate study results: (1) at conferences (scientific and professional) whose themes relate to SDM, IP and health policy; (2) on the websites of team members and (3) as articles in peerreviewed journals and professional journals intended for policymakers and managers (eg, the Ministère de la Santé et des Services sociaux bulletin Coup d'œil). Furthermore, skills gained by home care teams in this study are likely to be transferable to support clients who are making other decisions, such as those related to mental health.

\section{Trial status}

Participant recruitment started on November 2015 and we anticipate it will be complete by June 2018 .

\section{Author affiliations}

${ }^{1} \mathrm{CHU}$ de Québec Research Centre, Saint-François d'Assise Hospital, Quebec City, Quebec, Canada

${ }^{2}$ Faculty of Medicine, Université Laval, Quebec City, Quebec, Canada ${ }^{3}$ Centre intégré universitaire de santé et de services sociaux (CIUSSS) de la Capitale-Nationale, Direction des services multidisciplinaires, Quebec City, Quebec, Canada

${ }^{4}$ Clinical Epidemiology Program, Ottawa Hospital Research Institute, Ottawa, Ontario, Canada

${ }^{5}$ School of Nursing, University of Ottawa, Ottawa, Ontario, Canada

${ }^{6}$ Faculty of Social Sciences, Université Laval, Quebec City, Quebec, Canada

${ }^{7}$ School of Nutrition, Université Laval, Quebec City, Quebec, Canada

${ }^{8}$ Centre intégré universitaire de santé et de services sociaux (CIUSSS) de la

Capitale-Nationale, CERSSPL-UL, Quebec City, Quebec, Canada

${ }^{9}$ Faculty of Nursing, University of Alberta, Edmonton, Alberta, Canada

${ }^{10}$ Faculty of Sciences and Engineering, Université Laval, Quebec City, Quebec, Canada

${ }^{11}$ School of Epidemiology, University of Ottawa, Ottawa, Ontario, Canada

${ }^{12}$ Caregivers' representative, CHU de Québec Research Centre, Saint-François d'Assise Hospital, Quebec City, Quebec, Canada

Acknowledgements FL is supported by a Tier 1 Canada Research Chair. DS is supported by a Research Chair at the University of Ottawa. SDe is supported by a New Investigator Salary Award from the Canadian Institutes of Health Research (CIHR). L-PR is supported by a Tier 1 Canada Research Chair GL is supported by the Industrial Alliance Research Chair on the Economics of Population Ageing.

Contributors FL, NB and DS conceived the study. GL provided guidance for the planned cost-effectiveness analyses. SDe, SDu and KDF provided guidance on the Interprofessional Shared Decision-making Model and the training workshop materials. L-PR, MT and ST provided guidance for the planned statistical analyses. PJD, HB and LR validated the relevance of the trial and provided guidance on the methods. GPG is coordinating the project. FL and GPG drafted the manuscript. All authors have read and approved the final version of the manuscript. $F L$ is its guarantor.

Funding This research is funded by the Canadian Institutes of Health Research (Grant number: 201403MOP-325236-KTR-CFBA-19158), and also supported by the CIUSSS de la Capitale-Nationale (in kind contribution included in the CIHR grant).

Competing interests DS reports personal fees from Washington State Health Authority Patient Decision Aid certification programme, outside the submitted work.
Patient consent Obtained.

Ethics approval Centre intégré de santé et de services sociaux de Laval (CISSS de Laval).

Provenance and peer review Not commissioned; externally peer reviewed.

Open Access This is an Open Access article distributed in accordance with the Creative Commons Attribution Non Commercial (CC BY-NC 4.0) license, which permits others to distribute, remix, adapt, build upon this work noncommercially, and license their derivative works on different terms, provided the original work is properly cited and the use is non-commercial. See: http:// creativecommons.org/licenses/by-nc/4.0/

\section{REFERENCES}

1. Canadian Institute for Health Information. Health care in Canada, 2011: a focus on seniors and aging. Ottawa, ON: 2011. https:// secure.cihi.ca/free_products/HCIC_2011_seniors_report_en.pdf (accessed 16 Nov 2016).

2. Déoux $S$, Massot $O$, Girard V. L'habitat, facteur de santé des trente dernières années de vie des aînés. Gérontol Soc 2011;136:91-103.

3. Idler EL, Benyamini Y. Self-rated health and mortality: a review of twenty-seven community studies. J Health Soc Behav 1997;38:21-37.

4. Ramage-Morin PL. Successful aging in health care institutions. Health Rep 2006;16:47-56.

5. Gaugler JE, Pearlin LI, Leitsch SA, et al. Relinquishing in-home dementia care: difficulties and perceived helpfulness during the nursing home transition. Am J Alzheimers Dis Other Demen 2001;16:32-42.

6. Barnes M, Bennett G. Frail bodies, courageous voices: older people influencing community care. Health Soc Care Community 1998:6:102-11.

7. Caron CD, Ducharme F, Griffith J. Deciding on institutionalization for a relative with dementia: the most difficult decision for caregivers. Can J Aging 2006;25:193-205.

8. Gaugler JE, Zarit SH, Pearlin LI. Caregiving and institutionalization: perceptions of family conflict and socioemotional support. Int J Aging Hum Dev 1999;49:1-25.

9. Ryan AA, Scullion HF. Nursing home placement: an exploration of the experiences of family carers. J Adv Nurs 2000;32:1187-95.

10. Stacey D, Légaré $F$, Col NF, et al. Decision aids for people facing health treatment or screening decisions. Cochrane Database Syst Rev 2014;(1):CD001431.

11. Légaré F, O'Connor AM, Graham ID, et al. The effect of decision aids on the agreement between women's and physicians' decisional conflict about hormone replacement therapy. Patient Educ Couns 2003:50:211-21.

12. Légaré $\mathrm{F}$, Stacey $\mathrm{D}$, Gagnon $\mathrm{S}$, et al. Validating a conceptual model for an inter-professional approach to shared decision making: a mixed methods study. J Eval Clin Pract 2011:17:554-64.

13. Schottenfeld L, Petersen D, Peikes D, et al. Creating patientcentered team-based primary care. Rockville: Agency for Healthcare Research and Quality, 2016.

14. Hofstede SN, Marang-van de Mheen PJ, Wentink MM, et al. Barriers and facilitators to implement shared decision making in multidisciplinary sciatica care: a qualitative study. Implement Sci 2013;8:95

15. Giguère $\mathrm{A}$, Légaré $\mathrm{F}$, Grimshaw $\mathrm{J}$, et al. Printed educational materials: effects on professional practice and healthcare outcomes. Cochrane Database Syst Rev 2012;10:CD004398.

16. Brown CA, Lilford RJ. The stepped wedge trial design: a systematic review. BMC Med Res Methodol 2006;6:54.

17. Santé et Services sociaux Québec. Network reorganization. Quebec government, 2015. http://www.msss.gouv.qc.ca/en/reseau/ reorganisation/portrait (accessed 23 Aug 2016).

18. Légaré $\mathrm{F}$, Brière $\mathrm{N}$, Stacey $\mathrm{D}$, et al. Improving Decision making On Location of Care with the frail Elderly and their caregivers (the DOLCE study): study protocol for a cluster randomized controlled trial. Trials 2015;16:50.

19. Stacey $D$, Higuchi KA, Menard $P$, et al. Integrating patient decision support in an undergraduate nursing curriculum: an implementation project. Int J Nurs Educ Scholarsh 2009;6:Article10.

20. O'Connor AM, Tugwell P, Wells GA, et al. A decision aid for women considering hormone therapy after menopause: decision support framework and evaluation. Patient Educ Couns 1998;33: 267-79.

21. Stacey $\mathrm{D}$, Brière $\mathrm{N}$, Robitaille $\mathrm{H}$, et al. A systematic process for creating and appraising clinical vignettes to illustrate 
interprofessional shared decision making. $J$ Interprof Care 2014;28:453-9.

22. Strull WM, Lo B, Charles G. Do patients want to participate in medical decision making? JAMA 1984;252:2990-4.

23. Degner LF, Sloan JA. Decision making during serious illness: what role do patients really want to play? J Clin Epidemiol 1992;45:941-50.

24. Légaré F, Turcotte S, Stacey D, et al. Patients' perceptions of sharing in decisions: a systematic review of interventions to enhance shared decision making in routine clinical practice. Patient 2012;5:1-19.

25. Melbourne E, Sinclair K, Durand MA, et al. Developing a dyadic OPTION scale to measure perceptions of shared decision making. Patient Educ Counsel 2010;78:177-83.

26. Melbourne E, Roberts S, Durand MA, et al. Dyadic OPTION: measuring perceptions of shared decision-making in practice. Patient Educ Counsel 2011;83:55-7.

27. O'Connor AM. Validation of a decisional conflict scale. Med Decis Making 1995;15:25-30.

28. Légaré $\mathrm{F}$, Graham ID, O'Connor AM, et al. Prise de décision pargagée: traduction et validation d'une échelle de confort décisionnel du médecin. Pédagogie médicale 2003;4:216-22.

29. Brehaut JC, O'Connor AM, Wood TJ, et al. Validation of a decision regret scale. Med Decis Making 2003;23:281-92.

30. Faria CD, Teixeira-Salmela LF, Nascimento VB, et al. Comparisons between the Nottingham Health Profile and the Short Form-36 for assessing the quality of life of community-dwelling elderly. Rev Bras Fisioter 2011;15:399-405.

31. Sharples L, Todd C, Caine N, et al. Measurement properties of the Nottingham Health Profile and Short Form 36 health status measures in a population sample of elderly people living at home: results from ELPHS. Br J Health Psychol 2000;5:217-33.

32. Zengin N, Ören B, Gül A, et al. Assessment of quality of life in haemodialysis patients: a comparison of the Nottingham Health Profile and the Short Form 36. Int J Nurs Pract 2014;20:115-25.

33. Bucquet D, Condon S, Ritchie K. The French version of the Nottingham Health Profile. A comparison of items weights with those of the source version. Soc Sci Med 1990;30:829-35.
34. Zarit SH, Orr NK, Zarit JM. The hidden victims of Alzheimer's disease: families under stress. New York: New York University Press, 1985.

35. Seng BK, Luo N, Ng WY, et al. Validity and reliability of the Zarit Burden Interview in assessing caregiving burden. Ann Acad Med Singap 2010;39:758-63.

36. Hébert R, Bravo G, Girouard D. Fidélité de la traduction française de trois instruments d'évaluation des aidants naturels de malades déments. Can J Aging 1993;12:324-37.

37. Légaré $F$, Borduas $F$, Freitas $A$, et al. Development of a simple 12 -item theory-based instrument to assess the impact of continuing professional development on clinical behavioral intentions. PIOS ONE 2014:9:e91013.

38. Hussey MA, Hughes JP. Design and analysis of stepped wedge cluster randomized trials. Contemp Clin Trials 2007;28:182-91.

39. Woertman W, de Hoop E, Moerbeek M, et al. Stepped wedge designs could reduce the required sample size in cluster randomized trials. J Clin Epidemiol 2013;66:752-8.

40. Zou G, Donner A. Confidence interval estimation of the intraclass correlation coefficient for binary outcome data. Biometrics 2004:60:807-11.

41. Adams G, Gulliford MC, Ukoumunne OC, et al. Patterns of intra-cluster correlation from primary care research to inform study design and analysis. J Clin Epidemiol 2004;57:785-94.

42. Campbell MK, Fayers PM, Grimshaw JM. Determinants of the intracluster correlation coefficient in cluster randomized trials: the case of implementation research. Clin Trials 2005;2:99-107.

43. Ukoumunne OC, Gulliford MC, Chinn S, et al. Methods for evaluating area-wide and organisation-based interventions in health and health care: a systematic review. Health Technol Assess 1999;3:iii-92.

44. Smeeth L, Ng ES. Intraclass correlation coefficients for cluster randomized trials in primary care: data from the MRC Trial of the Assessment and Management of Older People in the Community. Control Clin Trials 2002:23:409-21.

45. Couët N, Desroches S, Robitaille H, et al. Assessments of the extent to which health-care providers involve patients in decision making: a systematic review of studies using the OPTION instrument. Health Expect 2015;18:542-61. 